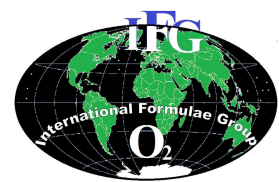

Available online at http://ajol.info/index.php/ijbcs

Int. J. Biol. Chem. Sci. 8(4): 1710-1729, August 2014

International Journal

of Biological and

Chemical Sciences

ISSN 1997-342X (Online), ISSN 1991-8631 (Print)

Original Paper

http://indexmedicus.afro.who.int

\title{
Diversité agro-morphologique des accessions de fonio [Digitaria exilis (Kippist.) Stapf.] au Niger
}

\author{
Sani IDI SAIDOU ${ }^{1^{*}}$, Yacoubou BAKASSO ${ }^{1}$, Maman Maarouhi INOUSSA ${ }^{1}$, \\ Mainassara ZAMAN-ALLAH ${ }^{2}$, Sanoussi ATTA ${ }^{3}$, Adeline BARNAUD ${ }^{4,5}$, \\ Claire BILLOT ${ }^{6}$ et Mahamane SAADOU ${ }^{1,2}$ \\ ${ }^{1}$ Université Abdou Moumouni de Niamey, Faculté des Sciences et Techniques, Département de Biologie, \\ Laboratoire Garba Mounkaila. BP: 10662, Niamey, Niger. \\ ${ }^{2}$ Université de Maradi, Faculté des Sciences et Techniques, Département de Biologie. \\ BP : 465, Maradi, Niger. \\ ${ }^{3}$ Centre Régional AGRYMET, Département de Formation et de Recherche, BP. 12625 Niamey, Niger. \\ ${ }^{4}$ Institut de Recherche pour le Développement, UMR DIADE, 911, \\ avenue Agropolis - BP 6450134394 Montpellier cedex 5, France. \\ ${ }^{5}$ Laboratoire mixte international Adaptation des Plantes et microorganismes associés aux Stress \\ Environnementaux (LMI LAPSE), Institut de Recherche pour le Développement/Institut Sénégalais de \\ Recherches Agricoles/Université Cheikh Anta Diop, Centre de Recherche de Bel Air, Dakar, Sénégal. \\ ${ }^{6}$ Cirad, UCentre International de Recherche Agronomique pour le Développement, UMR-AGAP. A A-108/03, \\ Avenue d'Agropolis F-34398 Montpellier Cedex 5, Montpellier, France. \\ *Auteur correspondant ; E-mail: saidousani@yahoo.fr; ssaidouidi@gmail.com ; \\ Tel : $0022790613650 ; 0022789562367$
}

\section{RÉSUMÉ}

La variablité morphologique de 67 accessions de fonio [Digitaria exilis (Kippist.) Stapf.] collectées au Niger a été évaluée au cours de deux années (2011 et 2012). Seize (16) caractères agro morphologiques (dont 14 caractères quantitatifs et 2 caractères qualitatifs) ont été évalués en station dans deux zones agroécologiques différentes (Tarna en zone sahélo-saharienne et Tara en zone soudanienne). La classification acsendante hierarchique (CAH) et l'analyse factorielle discriminante (AFD) ont mis en évidence quatre groupes (GI, GII, GIII et GIV). Les accessions du groupe GIV ont en moyenne un cycle de maturité compris entre 85 et 90 jours tandis que les autres groupes présentent un cycle de maturité superieur à 90 jours dans nos conditions expérimentales. Les résultats montrent que les variables les plus discriminantes qui permettent de décrire la variabilité entre les groupes identifiés sont la biomasse sèche (tige et feuille) par hectare, la longueur des entrenœuds, le rendement en graine par hectare et dans une certaine mesure le cycle de la plante. L'observation des caractères qualitatifs a montré que cinq accessions (représentant $7 \%$ du total) ont des graines non decortiquées de couleur rouge et quatre accessions ( $6 \%$ du total), une tige de couleur rouge. Les caractères analysés peuvent ainsi constituer des critères de base pour différencier les accessions des autres régions de l'Afrique de l'Ouest et servir pour une étude de variabilité entre les restes des accessions de fonio collectées au Niger. Des possiblités d'amélioration peuvent également être envisagées une fois que le regime de réproduction de l'espèce est bien identifié.

(c) 2014 International Formulae Group. All rights reserved.

Mots clés: Digitaria exilis, variabilité morphologique, accessions, diversité, fonio, Niger.

(C) 2014 International Formulae Group. All rights reserved. DOI : http://dx.doi.org/10.4314/ijbcs.v8i4.31 


\section{INTRODUCTION}

Les variétés traditionnelles des espèces céréalières cultivées sont en voie de disparition dans plusieurs régions du monde tropical. En effet, l'introduction des cultures de rente et de variétés améliorées à haut rendement a contribué à restreindre l'aire de culture des variétés traditionnelles qu'on peut retrouver dans les campagnes les plus reculées (Brush, 1995). Les études de diversité des plantes cultivées ont montré que les variétés traditionnelles, bien que moins productives, sont génétiquement plus diversifiées que les variétés améliorées (Brown et Munday, 1982 ; Ahmadi et al., 1988). Par ailleurs, la sauvegarde de ces variétés traditionnelles contribue au maintien de leur richesse génétique (Frankel et al., 1995) et permet de conserver le potentiel nécesaire pour faire face à de nouveaux impératifs (Cherifi et al., 1993). Pour éviter une disparition considérée comme une catastrophe biologique, de nombreuses accessions de variétés traditionnelles ont été récoltées et conservées dans des banques de gènes (Harlan, 1975; Frankel et al., 1995; Cruz, 2004). Ces accessions maintenues et reproduites ex situ ont servi de matériel biologique dans de nombreuses études de caractérisation morphologique (Chantereau et al., 1989; Adoukounou et al., 2007b), enzymatique (Maquet et al., 1997, Adoukounou et al., 2007a) et moléculaire (Fofana et al., 1997; Adoukounou, 2010). Cependant, en raison de biais d'échantillonnage et d'une longue phase de conservation, la variabilité génétique observée notamment en termes d'associations d'allèles ne reflète plus toujours la diversité actuellement présente in situ (Doebley et al., 1985 ; Djè et al., 1998). Il convient dans la mesure du possible, de porter un regard nouveau sur le matériel végétal dans son aire naturelle de culture, surtout en ce qui concerne les espèces cultivées. Cela est urgent pour les études morphologiques car les réproductions en serres, même tropicalisées, n'offrent jamais les mêmes conditions que celles rencontrées dans les pays d'origine.

Le fonio [Digitaria exilis (Kippist.) Stapf.] est une herbacée monoïque annuelle de 30 à $80 \mathrm{~cm}$ de hauteur présentant une inflorescence composée le plus souvent de deux ou trois racèmes ou épis (Portères, 1946; Poilecot, 1999). Bien adapté aux conditions édaphiques et climatiques locales, il résiste à la sécheresse grâce à son métabolisme en $\mathrm{C} 4$ et participe au maintien de l'environnement en assurant une couverture végétale sur des sols écologiquement fragiles et peu valorisés (Cruz, 2004; Cruz et al., 2011 ; Vall et al., 2011). Il semble moins sensible aux dommages parasitaires pendant le stockage que d'autres céréales (Dansi et al., 2010; Adoukonou-Sagbadja et al., 2007b). Les grains de fonio sont consommés sous diverses formes (couscous, bouillies) et les pailles sont souvent utilisées comme fourrage pour nourrir les bovins, mais aussi les chèvres, les moutons notamment dans les zones les plus arides, où les sources d'alimentation animale sont rares (Cruz, 2004 ; Ezekiel et al., 2012; Jideani et Jideani, 2010; Jideani, 2012). Pauvre en gluten, le fonio est indiqué pour les personnes diabétiques et ceux qui souffrent du problème de surpoids (Bama, 1999, Vodouhè et al., 2006).

$\mathrm{Au}$ Niger, les principales zones de production du fonio se trouvent dans les régions de Dosso et Tillabéry. Cette culture qui a longtemps été négligée connaît de nos jours un regain d'intérêt grâce à sa forte contribution dans la lutte contre l'insécurité alimentaire au Niger (Oumarou, 2012).

Cette étude se propose d'évaluer la diversité des accessions locales de fonio dans les deux principales zones de production du Niger. Spécifiquement, il s'agit (i) : d'analyser la variabilité agromorphologiques des accessions de fonio du Niger; et (ii) : d'identifier les variables les plus 
discriminantes qui différencient ces accessions.

\section{MATÉRIEL ET MÉTHODES}

\section{Sites d'étude}

Les essais ont été conduits pendant les campagnes agricole 2011 et 2012 dans deux stations de recherche de l'Institut National de la Recherche Agronomique du Niger (INRAN), la station de Tarna à Maradi (latitude $\mathrm{N} 13^{\circ} 28^{\prime}$ et longitude $\mathrm{E} 7^{\circ} 05^{\prime}$ ) située dans la zone sahelienne à environ $670 \mathrm{~km}$ au Sud Est de Niamey, et la station de Tara à Gaya (latitude N11 ${ }^{\circ} 53^{\prime}$ et longitude E3 ${ }^{\circ} 19^{\prime}$ ) située dans la zone soudanienne, à environ $300 \mathrm{~km}$ au Sud de Niamey. La longueur moyenne de la saison des pluies est de 120 à 146 jours à Gaya et 80 à 100 jours à Maradi (Sivakumar et al. 1993). En 2011 et 2012, les cumuls pluviométriques ont été respectivement de $475,7 \mathrm{~mm}$ en 37 jours et $510,7 \mathrm{~mm}$ en 40 jours à la station de Tarna et de $902,3 \mathrm{~mm}$ en 45 jours et $975,9 \mathrm{~mm}$ en 44 jours à la station de Tara. Au cours des essais, la température moyenne journalière a varié entre $22,5^{\circ} \mathrm{C}$ et $31,2^{\circ} \mathrm{C}$ en 2011 et, $22,6^{\circ} \mathrm{C}$ et $38,1^{\circ} \mathrm{C}$ en 2012 à Gaya. A Maradi, elle a varié entre $22,1^{\circ} \mathrm{C}$ et $37,5^{\circ} \mathrm{C}$ en 2011 , et entre $24,2^{\circ} \mathrm{C}$ et $40,1^{\circ} \mathrm{C}$ en 2012 . Les sols de la station de Tarna sont du type ferrugineux tropicaux profonds à $\mathrm{pH} 6$ et contenant plus de $96 \%$ de sables totaux. Leur teneur en matière organique est très faible $(0,22 \%)$, de même que leur teneur en azote total $(0,01 \%)$. La capacité d'echange cationique $(1,0$ meq/100g) est aussi faible avec une carence accusée en phosphore (1,66 ppm). Le sol de Tara est également de type ferrugineux tropical avec un $\mathrm{pH}$ de 5,1 et contient plus de $85 \%$ de sables totaux. Sa teneur en matière organique est faible $(0,3 \%)$, de même que celle de l'azote total $(0,02 \%)$. Sa capacité d'echange cationique $(1,04 \mathrm{meq} / 100 \mathrm{~g})$ est faible avec cependant une carence en phosphore (101 ppm) moins accusée que celle de Tarna (Bakasso, 2010; Abdoulkader, 2012).

\section{Matériel végétal}

Le matériel végétal était composé de 67 accessions de fonio collectées au mois de juin 2011 dans les deux principales régions (Dosso et Tillabéry) de production du fonio au Niger (Figure 1, Tableau 1). Les semences prélevées des stocks semenciers d'un paysan constituent une accession.

\section{Méthodes}

Dispositif expérimental et conduite de l'essai

L'expérimentation a été conduite selon un dispositif en blocs de Fisher complètement randomisés avec trois répétitions. Dans chaque bloc, une accession est représentée par un carré d'une superficie de $1 \mathrm{~m}^{2}$. Les carrés d'un même bloc sont séparés d'un mètre les uns des autres et les blocs successifs de deux mètres.

Deux grammes de chaque accession ont été semés à la volée par parcelle. Les semis ont été effectués les 4 et 9 juillet respectivement à Tara et Tarna pendant les saisons pluvieuses 2011 et 2012 (Figure 1). Un premier désherbage manuel a été effectué seize jours après semis (JAS), suivis de trois autres afin de limiter la compétition des plantes avec les mauvaises herbes. De plus, un apport de fumure sous forme de NPK, à la dose de $10 \mathrm{~g} / \mathrm{m}^{2}$, a été réalisé les trente septième $\left(37^{\text {ième }}\right)$ jours après le semis.

\section{Observation des caractères}

Deux catégories de caractères ont été évaluées. Il s'agit de 14 caractères quantitatifs décrits dans le Tableau 2 et deux caractères qualitatifs, la couleur de la graine non décortiquée (CGD) et la couleur de la tige (CTG).

\section{Analyse des données}

Les données obtenues ont été analysées 
Windows, JMP9 (SAS, 2010) et R.2.15 for Windows. Les performances des différentes accessions ont été évaluées en déterminant pour chaque caractère quantitatif la moyenne, l'écart type, le coefficient de variation, le minimum et le maximum. Après l'analyse de la normalité par le test de Ryan-Joiner et de l'égalité des variances par le test de Levene, une analyse de variance (ANOVA) basée sur la comparaison des moyennes multiples a également été realisée sur les differents caractères quantitatifs. Par ailleurs, le degré d'association entre les différents caractères étudiés a été estimé par le coefficient de corrélation de Pearson. Afin d'apprécier le degré de ressemblance entre les unités analysées et comprendre les relations existantes entre elles, les données recueillies ont été soumises à une classification ascendante hiérarchique (CAH) basée sur la distance euclidienne selon la méthode de Ward et une analyse factorielle discriminante (AFD). Enfin, le test t de Student-NewmanKeuls a été utilisé pour comparer les moyennes des paramètres par groupe et par accessions deux à deux.

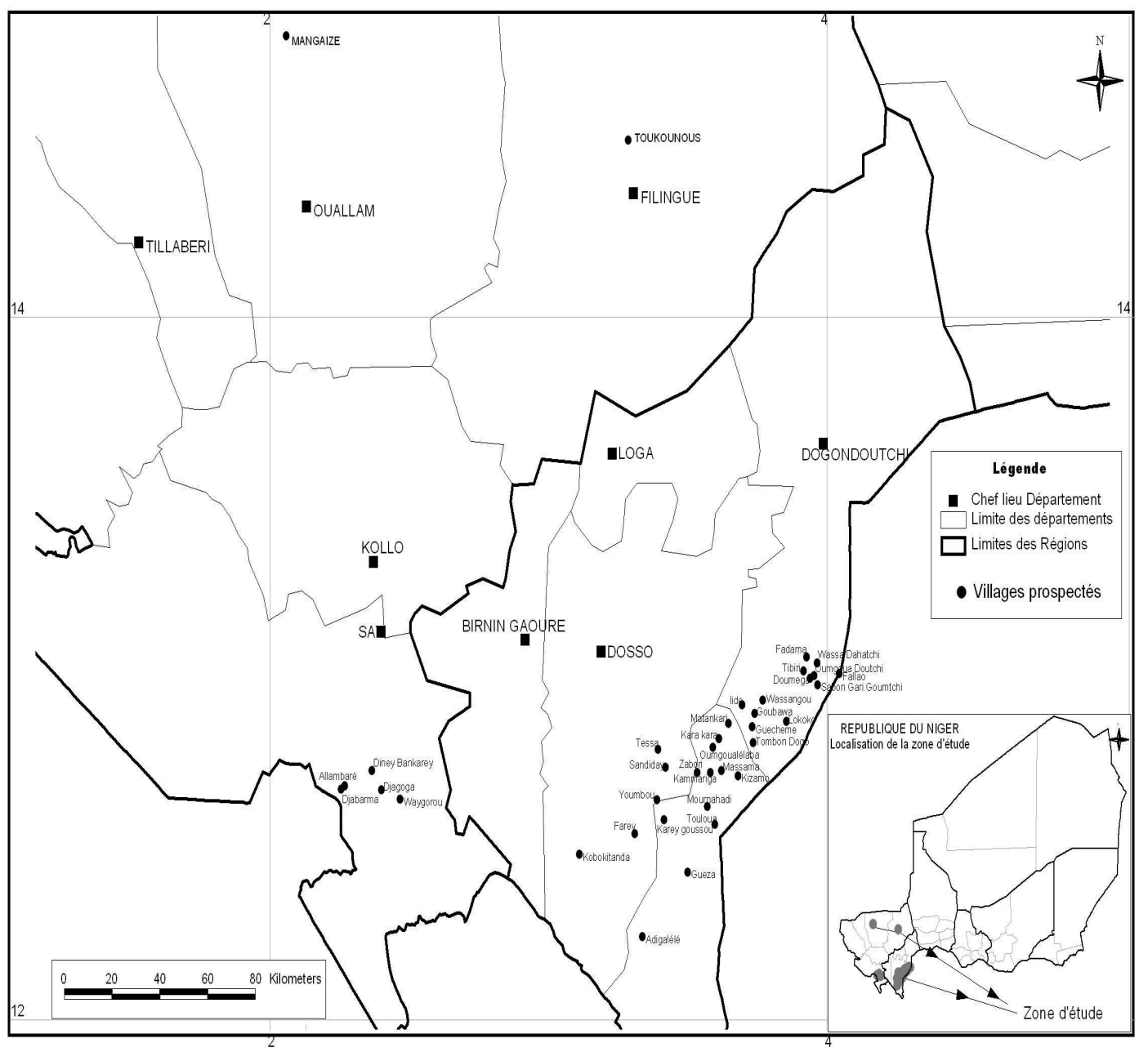

Figure 1: Situation géographique des villages prospectés pour la culture du fonio au Niger. 


\section{S. I. SAIDOU et al. /Int. J. Biol. Chem. Sci. 8(4): 1710-1729, 2014}

Tableau 1: Informations relatives aux sites de prospection des accessions du fonio collectées en 2011 au Niger.

\begin{tabular}{|c|c|c|c|c|c|c|c|c|c|c|c|}
\hline Accession & Régions & Communes & Villages & Latitude & Longitude & Accession & Régions & Communes & Villages & Latitude & Longitude \\
\hline Ech184 & Dosso & Dioundou & Karey goussou & $\mathrm{N} 12^{\circ} 34^{\prime} 03.5^{\prime \prime}$ & E0032'ㄷ‥1" & Ech153 & Dosso & Kara kara & Massama & $\mathrm{N} 12^{\circ} 42^{\prime 2} 27.1^{\prime \prime}$ & E0033'ㄷ‥' \\
\hline Ech194 & Dosso & Dioundou & Mournahadi & N12 $36^{\prime} 19.4^{\prime \prime}$ & E003ํำ'36.9" & Ech154 & Dosso & Kara kara & Massama & $\mathrm{N} 12^{\circ} 42^{\prime} 27.1^{\prime \prime}$ & E0033'ㄷ‥ \\
\hline Ech196 & Dosso & Dioundou & Mournahadi & N12 $36^{\prime} 19.4^{\prime \prime}$ & 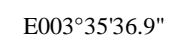 & Ech151 & Dosso & Kara kara & Oumgoualélaba & $\mathrm{N} 12^{\circ} 46^{\prime} 28.7^{\prime \prime}$ & E0033'ㄷ‥ \\
\hline Ech189 & Dosso & Dioundou & Touloua & N12 $33^{\prime} 16.5^{\prime \prime}$ & E0033'ㄴ․ & Ech152 & Dosso & Kara kara & Oumgoualélaba & N12 $46^{\prime} 28.7^{\prime \prime}$ & E0033'ㄷ‥ \\
\hline Ech190 & Dosso & Dioundou & Touloua & N12 $33^{\prime} 16.5^{\prime \prime}$ & E0033'ㄴ․ 24. & Ech125 & Dosso & Matankari & Matankari & $\mathrm{N} 12^{\circ} 50^{\prime} 32.8^{\prime \prime}$ & E003ํํ'.25.1" \\
\hline Ech192 & Dosso & Dioundou & Touloua & $\mathrm{N} 12^{\circ} 33^{\prime} 16.5^{\prime \prime}$ & E0033'ㄴ․ 24. & Ech127 & Dosso & Matankari & Matankari & $\mathrm{N} 12^{\circ} 50^{\prime} 32.8^{\prime \prime}$ & E003ㄴㅇ'.25.1" \\
\hline Ech179 & Dosso & Dioundou & Youmbou & $\mathrm{N} 12^{\circ} 37^{\prime} 32.8^{\prime \prime}$ & $\mathrm{E} 003^{\circ} 24^{\prime} 18.6^{\prime \prime}$ & Ech128 & Dosso & Matankari & Matankari & $\mathrm{N} 12^{\circ} 50^{\prime} 32.8^{\prime \prime}$ & E003ํํ'.25.1" \\
\hline Ech180 & Dosso & Dioundou & Youmbou & $\mathrm{N} 12^{\circ} 37^{\prime} 32.8^{\prime \prime}$ & $\mathrm{E} 003^{\circ} 24^{\prime} 18.6^{\prime \prime}$ & Ech174 & Dosso & Tessa & Sandiday & $\mathrm{N} 12^{\circ} 43^{\prime} 07.1^{\prime \prime}$ & $\mathrm{E} 003^{\circ} 26^{\prime} 12.5^{\prime \prime}$ \\
\hline Ech181 & Dosso & Dioundou & Youmbou & $\mathrm{N} 12^{\circ} 37^{\prime} 32.8^{\prime \prime}$ & $\mathrm{E} 003^{\circ} 24^{\prime} 18.6^{\prime \prime}$ & Ech177 & Dosso & Tessa & Sandiday & $\mathrm{N} 12^{\circ} 43^{\prime} 07.1^{\prime \prime}$ & $\mathrm{E} 003^{\circ} 26^{\prime} 12.5^{\prime \prime}$ \\
\hline Ech8 & Dosso & Doumega & Doumega & N1258'19.6" & E0035'ㄷ․ & Ech170 & Dosso & Tessa & Tessa & $\mathrm{N} 12^{\circ} 46^{\prime} 07.3^{\prime \prime}$ & E0032ㄴ $33.2^{\prime \prime}$ \\
\hline Ech11 & Dosso & Doumega & Doumega & N1258'19.6" & E0035'ㄷ․ & Ech45 & Dosso & Tibiri & Birni Fallao & N12 $62^{\prime} 05.3^{\prime \prime}$ & 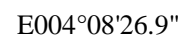 \\
\hline Ech214 & Dosso & Farrey & Farey & $\mathrm{N} 12^{\circ} 31^{\prime} 44.3^{\prime \prime}$ & $\mathrm{E}^{2} 03^{\circ} 19^{\prime} 17.1^{\prime \prime}$ & Ech46 & Dosso & Tibiri & Birni Fallao & $\mathrm{N} 12^{\circ} 62^{\prime} 05.3^{\prime \prime}$ & 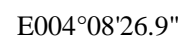 \\
\hline $\operatorname{Ech} 211$ & Dosso & Farrey & Farey & $\mathrm{N} 12^{\circ} 31^{\prime} 44.3^{\prime \prime}$ & $\mathrm{E} 003^{\circ} 19^{\prime} 17.1^{\prime \prime}$ & Ech44 & Dosso & Tibiri & Birni Fallao & $\mathrm{N} 13^{\circ} 00^{\prime} 40.8^{\prime \prime}$ & E004 $04^{\prime} 31.4^{\prime \prime}$ \\
\hline Ech77 & Dosso & Guecheme & Goubawa & N1252'14.6" & E0034' $19.6^{\prime \prime}$ & Ech23 & Dosso & Tibiri & $\begin{array}{c}\text { Oumgoua } \\
\text { Doutchi }\end{array}$ & $\mathrm{N} 12^{\circ} 58^{\prime} 47.3^{\prime \prime}$ & E003ㄷ'ㄴㄷ.3" \\
\hline Ech78 & Dosso & Guecheme & Goubawa & N12²5'14.6" & E0034' 19.6" & Ech1 & Dosso & Tibiri & Tibiri & $\mathrm{N} 12^{\circ} 59^{\prime} 29.0^{\prime \prime}$ & E003ㄷํ'23.2" \\
\hline Ech91 & Dosso & Guecheme & Goubawa & N1252'14.6" & E0034' $19.6^{\prime \prime}$ & Ech3 & Dosso & Tibiri & Tibiri & $\mathrm{N} 12^{\circ} 59^{\prime} 29.0^{\prime \prime}$ & 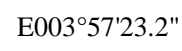 \\
\hline Ech238 & Dosso & Guecheme & Goubawa & N12 $52^{\prime} 14.6^{\prime \prime}$ & E0034' $19.6^{\prime \prime}$ & Ech204 & Dosso & Yelou & Adigalélé & $\mathrm{N} 12^{\circ} 14^{\prime} 08.2^{\prime \prime}$ & E00320'59.1" \\
\hline Ech95 & Dosso & Guecheme & Guecheme & N1249'56.9" & $\mathrm{E} 003^{\circ} 45^{\prime} 48.0^{\prime \prime}$ & Ech207 & Dosso & Yelou & Adigalélé & $\mathrm{N} 12^{\circ} 14^{\prime} 08.2^{\prime \prime}$ & $\mathrm{E}^{\circ}{ }^{\circ} 20^{\prime} 59.1^{\prime \prime}$ \\
\hline
\end{tabular}


S. I. SAIDOU et al. / Int. J. Biol. Chem. Sci. 8(4): 1710-1729, 2014

\begin{tabular}{|c|c|c|c|c|c|c|c|c|c|c|c|}
\hline Ech101 & Dosso & Guecheme & Guecheme & $\mathrm{N} 12^{\circ} 49^{\prime} 56.9^{\prime \prime}$ & $\mathrm{E} 003^{\circ} 45^{\prime} 48.0^{\prime \prime}$ & Ech208 & Dosso & Yelou & Adigalélé & $\mathrm{N} 12^{\circ} 14^{\prime} 08.2^{\prime \prime}$ & $\mathrm{E}^{2} 003^{\circ} 20^{\prime} 59.1^{\prime}$ \\
\hline Ech117 & Dosso & Guecheme & Lido & $\mathrm{N} 12^{\circ} 53^{\prime} 42.2^{\prime \prime}$ & E003ㄴ3'29.0" & Ech200 & Dosso & Yelou & Gueza & $\mathrm{N} 12^{\circ} 25^{\prime} 11.1^{\prime \prime}$ & $\mathrm{E}^{\prime} 003^{\circ} 31^{\prime} 11.6^{\prime}$ \\
\hline Ech121 & Dosso & Guecheme & Lido & $\mathrm{N} 12^{\circ} 53^{\prime} 42.2^{\prime \prime}$ & E003ㄴ3'29.0" & Ech159 & Dosso & Zabori & Kammariga & $\mathrm{N} 12^{\circ} 42^{\prime} 11.7^{\prime \prime}$ & 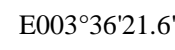 \\
\hline Ech60 & Dosso & Guecheme & Lokoko & $\mathrm{N} 12^{\circ} 50^{\prime} 52.0^{\prime \prime}$ & E0035'ㄹ. $27.0^{\prime \prime}$ & Ech160 & Dosso & Zabori & Kammariga & $\mathrm{N} 12^{\circ} 42^{\prime} 11.7^{\prime \prime}$ & 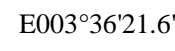 \\
\hline Ech67 & Dosso & Guecheme & Lokoko & $\mathrm{N} 12^{\circ} 50^{\prime} 52.0^{\prime \prime}$ & E0035'ㄹ․ & Ech162 & Dosso & Zabori & Kammariga & $\mathrm{N} 12^{\circ} 42^{\prime} 11.7^{\prime \prime}$ & $\mathrm{E}^{2} 03^{\circ} 36^{\prime} 21.6^{\prime}$ \\
\hline Ech104 & Dosso & Guecheme & $\begin{array}{c}\text { Tombon } \\
\text { Dogo }\end{array}$ & $\mathrm{N} 12^{\circ} 47^{\prime} 15.0^{\prime \prime}$ & E0034ㄷㄴㄷ.9" & Ech164 & Dosso & Zabori & Zabori & N12 $42^{\prime} 09.4^{\prime \prime}$ & 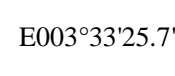 \\
\hline Ech70 & Dosso & Guecheme & Wassangou & $\mathrm{N} 12^{\circ} 54^{\prime} 29.3^{\prime \prime}$ & E0034'07.4" & Ech165 & Dosso & Zabori & Zabori & $\mathrm{N} 12^{\circ} 42^{\prime} 09.4^{\prime \prime}$ & $\mathrm{E}^{\prime} 03^{\circ} 33^{\prime} 25.7^{\prime}$ \\
\hline Ech71 & Dosso & Guecheme & Wassangou & $\mathrm{N} 12^{\circ} 54^{\prime} 29.3^{\prime \prime}$ & E003ㄴㅇ'07.4" & Ech224 & Tillabery & Tamou & Diney Bankarey & $\mathrm{N} 12^{\circ} 42^{\prime} 29.0^{\prime \prime}$ & $\mathrm{E} 002^{\circ} 20^{\prime} 07.0^{\prime}$ \\
\hline Ech72 & Dosso & Guecheme & Wassangou & N12 ${ }^{\circ} 54^{\prime} 29.3^{\prime \prime}$ & E003ㄴㅇ'07.4" & Ech236 & Tillabery & Tamou & Djabarma & $\mathrm{N} 12^{\circ} 39^{\prime} 17.8^{\prime \prime}$ & $\mathrm{E} 002^{\circ} 13^{\prime} 06.2^{\prime}$ \\
\hline Ech73 & Dosso & Guecheme & Wassangou & N1254'29.3" & E0034ㅇ'07.4" & Ech225 & Tillabery & Tamou & Djagoga & $\mathrm{N} 12^{\circ} 39^{\prime} 12.4^{\prime \prime}$ & $\mathrm{E} 002^{\circ} 22^{\prime} 14.1^{\prime}$ \\
\hline Ech74 & Dosso & Guecheme & Wassangou & N1254'29.3" & E003ㄴㅇ'07.4" & Ech227 & Tillabery & Tamou & Djagoga & N12 $39^{\prime} 12.4^{\prime \prime}$ & 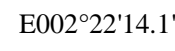 \\
\hline Ech68 & Dosso & Guecheme & Wassangou & N12 $54^{\circ} 29.3^{\prime \prime}$ & $\mathrm{E} 003^{\circ} 48^{\prime} 07.4^{\prime \prime}$ & Ech232 & Tillabery & Tamou & Waygorou & $\mathrm{N} 12^{\circ} 37^{\prime} 35.8^{\prime \prime}$ & $\mathrm{E} 002^{\circ} 26^{\prime} 23.4^{\prime}$ \\
\hline Ech146 & Dosso & Kara kara & Kara kara & $\mathrm{N} 12^{\circ} 47^{\prime} 55.5^{\prime \prime}$ & E0033'ㄷ․ & Ech234 & Tillabery & Tamou & Waygorou & N12 $37^{\prime} 35.8^{\prime \prime}$ & $\mathrm{E} 002^{\circ} 26^{\prime} 23.4^{\prime}$ \\
\hline Ech134 & Dosso & Kara kara & Kizamo & $\mathrm{N} 12^{\circ} 41^{\prime} 38.0^{\prime \prime}$ & E0034'ㄱ․ & & & & & & \\
\hline Ech136 & Dosso & Kara kara & Kizamo & $\mathrm{N} 12^{\circ} 41^{\prime} 38.0^{\prime \prime}$ & E003ㄴ2'33.9" & & & & & & \\
\hline Ech141 & Dosso & Kara kara & Kizamo & $\mathrm{N} 12^{\circ} 41^{\prime} 38.0^{\prime \prime}$ & 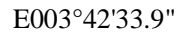 & & & & & & \\
\hline
\end{tabular}


Tableau 2: Variables quantitatives utilisées pour l'évaluation des accessions du fonio.

\begin{tabular}{|c|c|c|c|}
\hline Variables & Abréviations & Descriptions & Unité de mesure \\
\hline $\begin{array}{l}\text { Nombre de jours } 50 \% \text { émergence ou } \\
\text { levée }\end{array}$ & NJE & $\begin{array}{l}\text { Nombre de jours séparant le semis de la levée au stade } 2 \text { feuilles de } 50 \% \text { des graines } \\
\text { semées par accession. }\end{array}$ & jours \\
\hline Nombre de jours $50 \%$ Tallage & NJT & Nombre de jours séparant le semis du tallage de $50 \%$ des plantes par accession. & jours \\
\hline Nombre de talles par plante & NTP & $\begin{array}{l}\text { Moyenne sur } 5 \text { plantes du correspond au nombre de talles, représente le taux de tallage } \\
\text { d'une accession. }\end{array}$ & \\
\hline cycle de floraison & FLO & $\begin{array}{l}\text { Nombre de jours, du semis jusqu'au moment où } 50 \% \text { des plantes par } \mathrm{m}^{2} \text { ont fleuri, la } \\
\text { floraison étant considérée comme l'émergence du stigmate de l'épi principal. }\end{array}$ & jours \\
\hline cycle de maturité & MAT & $\begin{array}{l}\text { Nombre de jours, du semis jusqu'au moment où } 50 \% \text { des plantes par } \mathrm{m}^{2} \text { ont atteint leur } \\
\text { maturité. }\end{array}$ & jours \\
\hline hauteur de la plante à maturité & HPM & $\begin{array}{l}\text { Hauteur de la plante est mesurée en centimètre à partir du niveau du sol jusqu'au sommet de la } \\
\text { panicule sur } 5 \text { plantes par } \mathrm{m}^{2}\end{array}$ & $\mathrm{~cm}$ \\
\hline Longueur des entrenœuds & LEN & Mesuré entre le deuxième et dernier nœud de la plante situé au-dessous de la panicule. & $\mathrm{cm}$ \\
\hline Nombre d'entrenœuds & NEN & $\begin{array}{l}\text { Nombre des entre-nœuds par plante, la moyenne de } 5 \text { pieds par accession par } \mathrm{m}^{2} \text {. Il est obtenu sur } \\
\text { la tige principale et donne le nombre d'entre-nœuds extérieurs au sol. }\end{array}$ & \\
\hline Nombre des racèmes par panicule & NRP & Nombre des racèmes ou doigts par panicule, la moyenne de 5 pieds par accession par m². & \\
\hline Poids des graines par $\mathrm{m}^{2}$ & PGR & Mesure en $\mathrm{g}$ du poids des graines par $\mathrm{m}^{2} \mathrm{de}$ chaque accession après battage. & $\mathrm{g}$ \\
\hline Taille du racème par panicule & TRP & La longueur du doigts ou racème de la panicule mesurée sur 5 plantes par accession par m². & $\mathrm{cm}$ \\
\hline Longueur exertion de la panicule & EXE & $\begin{array}{l}\text { Mesure effectuée sur l'espace compris entre le début du doigt ou racème de la panicule et la fin } \\
\text { de la dernière gaine de la tige principale. Moyenne de } 5 \text { mesures faites sur des individus } \\
\text { différents du même accession. }\end{array}$ & $\mathrm{cm}$ \\
\hline Longueur des feuilles & $\mathrm{LoF}$ & $\begin{array}{l}\text { Mesure de la longueur de la troisième feuille de la tige principale située en dessous de la } \\
\text { panicule. Moyenne de } 5 \text { feuilles des individus du même accession. }\end{array}$ & $\mathrm{cm}$ \\
\hline Largeur des feuilles & $\mathrm{LaF}$ & $\begin{array}{l}\text { Mesure de la largeur de la troisième feuille de la tige principale. Moyenne de } 5 \text { feuilles des } \\
\text { individus du même accession. }\end{array}$ & $\mathrm{cm}$ \\
\hline Poids de la biomasse sèche par m² & PBM & $\begin{array}{l}\text { C'est le poids de la biomasse de chaque cultivar par } \mathrm{m}^{2} \text { séchée à } 65^{\circ} \mathrm{C} \text { à l'étuve pendant } 72 \\
\text { heures. }\end{array}$ & $\mathrm{g}$ \\
\hline $\begin{array}{l}\text { Groupement des épillets sur les } \\
\text { pédicelles au niveau des racèmes }\end{array}$ & GEP & épillets sur les pédicelles au niveau des racèmes de chaque panicule. & \\
\hline
\end{tabular}




\section{RÉSULTATS}

\section{Performance des accessions sur les caractères evalués}

Les caractères qualitatifs observés sur les accessions évaluées pendant les essais montrent que cinq accessions (7\% des accessions) et quatre (6\% accessions) présentent respectivement une couleur marron de grain non decortiqué (accessions : Ech19; Ech45; Ech74; Ech174 et Ech177) et une couleur rouge de la tige (accessions : Ech46; Ech192; Ech181 et Ech224).

Les résultats de la statistique descriptive indiquent des amplitudes de variation importantes (Tableau 3). Les coefficients de variation varient de $1,7 \%$ (Cycle de floraison et Cycle de maturité) à 27,9\% (Groupement des épillets sur les pédicelles au niveau des racèmes). On observe des valeurs significativement élevés (CV>20\%) pour un seul des 14 caractères métriques analysés (Tableau 3).

L'analyse de variance a montré que les accessions sont hautement significatives pour tous les caractères étudiés, à l'exception du nombre des jours $50 \%$ tallage (NJT), le nombre de talles par plante (NTP) et le nombre du racème par panicule (NRP). Les facteurs «Localités» et «Années » ne sont significatifs que pour les caractères cycle de floraison (FLO), cycle de maturité (MAT), nombre des jours $50 \%$ tallage (NJT), nombre de talles par plante (NTP), poids des grains par hectare (PGR) et poids de la biomasse sèche par hectare (PBM), à l'exception de la hauteur de la plante à maturité (HPM), le nombre du racème par panicule (NRP), le groupement des épillets sur les pédicelles au niveau des racèmes (GEP), l'exertion de la panicule (EXE), la longueur des feuilles (LoF), la largeur des feuilles (LaF), le nombre des entrenœuds (NEN) et la longueur des entrenœuds (LEN). L'effet bloc est significatif pour deux caractères : le nombre de jours $50 \%$ Tallage (NJT) et le nombre de talles par plante
(NTP). Par ailleurs, l'effet de l'interaction entre accession et localité d'une part, et accession et année d'essai d'autre part ont montré des differences hautement significatives pour sept caractères (PGR, GEP, EXE, LoF, LaF, NEN, LEN) alors que l'interaction entre accession et bloc d'essai au cour des deux campagnes d'évaluation n'est significative que pour la biomasse sèche par hectare (PBM) (Tableau 4).

\section{Corrélations entre les caractères étudiés}

Le Tableau 5 présente les corrélations entre les 14 caractères quantitatifs. Les corrélations significatives positives sont celles qui associent les caractères longueur des entrenoeuds (LEN) au poids de biomasse sèche par hectare (PBM) d'une part, et le caractère rendement en graine par hectare (PGR) au caractère nombre de talles (NTP) d'autre part avec $\mathrm{r}=0,5358, \mathrm{P}=0.021$. Cependant, il faut noter aussi les corrélations significatives négatives entre le rendement en graine par hectare (PGR) et la longueur des entrenoeuds (LEN) $(r=-0,5048, P=0.035)$ ou le poids de la biomasse sèche par hectare (PBM) $\quad(r=-0,8186, \quad P=0.002)$. Les corrélations négatives doivent attirer l'attention des sélectionneurs car lorsque deux caractères sont de corrélation négative, l'augmentation de l'un entraîne la diminution de l'autre. Ainsi, au regard de ces résultats, les plantes ayant le rendement le plus élevé en grain par hectare produisent une faible biomasse avec un nombre de talles plus élevé. A l'inverse, les plantes produisant une biomasse importante présentent un rendement moins élevé en grains.

\section{Structuration et groupement des accessions}

Le dendrogramme réalisé à partir des moyennes des 14 caractères quantitatifs basés sur la distance euclidienne selon la méthode de Ward a permis d'identifier quatre groupes (Figure 2). L'analyse factorielle discriminante 
(AFD) des distances de Mahalanobis (McLachlan, 1992), a permis de caractériser les 4 groupes obtenus sur la base de trois variables (PBM, PGR et LEN) (Tableau 6). Par ailleurs, l'examen des valeurs de la statistique $\mathrm{F}$ de Fisher et du coefficient de détermination $\mathrm{R}^{2}$ indique que les caractères PBM, PGR et LEN, sont les plus discriminants avec des valeurs de $\mathrm{F}$ et $\mathrm{R}^{2}$ relativement élevées. Ces résultats sont confirmés par la statistique de Wilks' Lambda $(\mathrm{P}<0,0001)$ mais aussi par le test Newmann Keuls (Tableau 8).

Les deux premiers axes canoniques concentrent à eux seuls presque la totalité $(98,04 \%)$ des informations contenues dans les variables initiales (Tableau 7). Le premier axe discriminant révèle l'opposition entre d'une part les valeurs de la biomasse sèche (PBM) et de longueur des entrenœuds et d'autre part le poids des graines (PGR). Cet axe est défini comme étant l'axe de rendement (biomasse ou grains) des accessions du fonio. En effet, de façon générale, les accessions d'un groupe à valeurs élevées de la biomasse sèche et de la longueur des entrenœuds ont de faibles valeurs du poids des graines (Tableau 8). Ainsi, toutes les accessions des groupes qui sont corrélées négativement à l'axe sont celles qui ont enregistré des fortes valeurs de la biomasse sèche. Ainsi, ces accessions ont donné des rendements très faibles en graine contrairement à celles des groupes corrélés positivement à l'axe 1 (Figures 3 et 4).

La projection des groupes dans le système d'axes canoniques 1 et 2 (Figure 3 ) indique que seul le premier axe canonique a un pouvoir discriminatif $(94,01 \%)$. Ainsi, les individus du groupe GIV possèdent des valeurs élevées de PGR, NTP, NEN, LoF et LaF alors que ceux du groupe GIII sont plus performants pour les paramètres tels que la biomasse sèche (PBM), la hauteur des plants à maturité (HPM), la phénologie (FLO et MAT), le nombre de jours $50 \%$ tallage (NJT), la longueur des entrenœuds (LEN), l'exertion de la panicule (EXE) et le nombre des racèmes par panicule (NRP). Les groupes GI et GII sont de performance moyenne pour l'ensemble des caractères; toutefois, les valeurs de HPM, NRP, NJT, EXE et MAT du groupe GI sont relativement plus élevées que celles du groupe GII. De même, les accessions du groupe GII possèdent de valeurs plus élevées en NTP et PGR que celles du groupe GIII.

Les accessions des quatre groupes possèdent la même performance pour le groupement des épillets sur les pédicelles au niveau des racèmes (GEP) (Tableau 8). Mais les accessions du groupe GIV sont caractérisées par un rendement plus important en graines à l'hectare (PGR) et sont beaucoup plus précoces que celles du groupe GIII. Ces dernières sont caractérisées par un rendement plus important en biomasse sèche à l'hectare (PBM) et sont relativement plus tardives que les accessions du groupe GII.

\section{DISCUSSION}

L'évaluation des caractères qualitatifs faite sur les accessions du fonio du Niger a permis d'observer une prédominance d'un type morphologique. Ainsi, 93\% des individus ont des graines non decortiquées de couleur jaune pâle (CGD), 94\% possèdent des tiges de couleur rouge (CTG) et enfin $7 \%$ et $6 \%$ ont respectivement une couleur marron des graines non décortiquées (CGD) et une couleur rouge de la tige (CTG).

La tendance à la prédominance d'un type morphologique donné, pour le caractère couleur de la tige est observée au niveau des groupes GII et GIV à l'exception notable de la couleur des graines non decortiquées. 


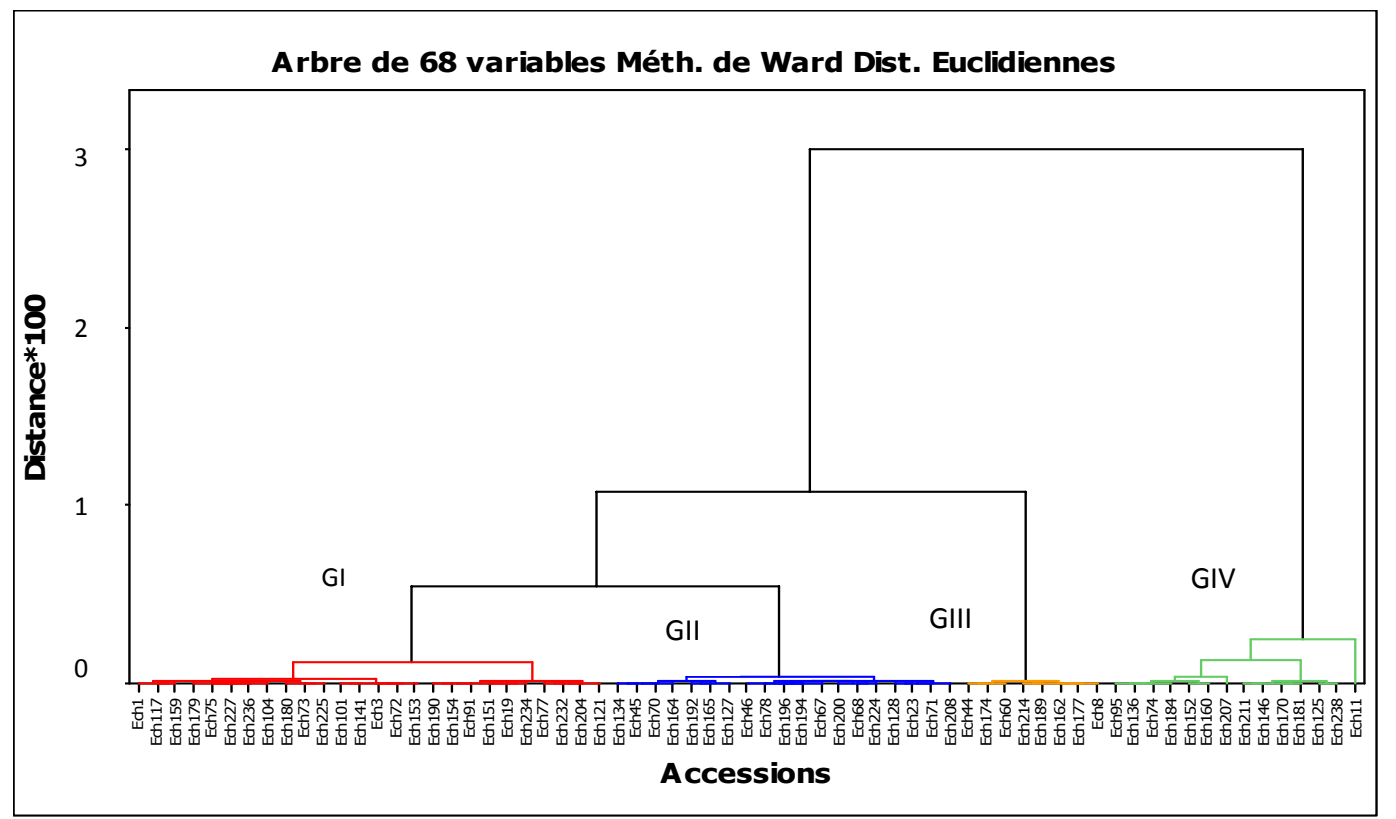

Figure 2 : Dendrogramme issu de la CAH des accessions de Digitaria exilis (Kippist.) Stapf. du Niger.

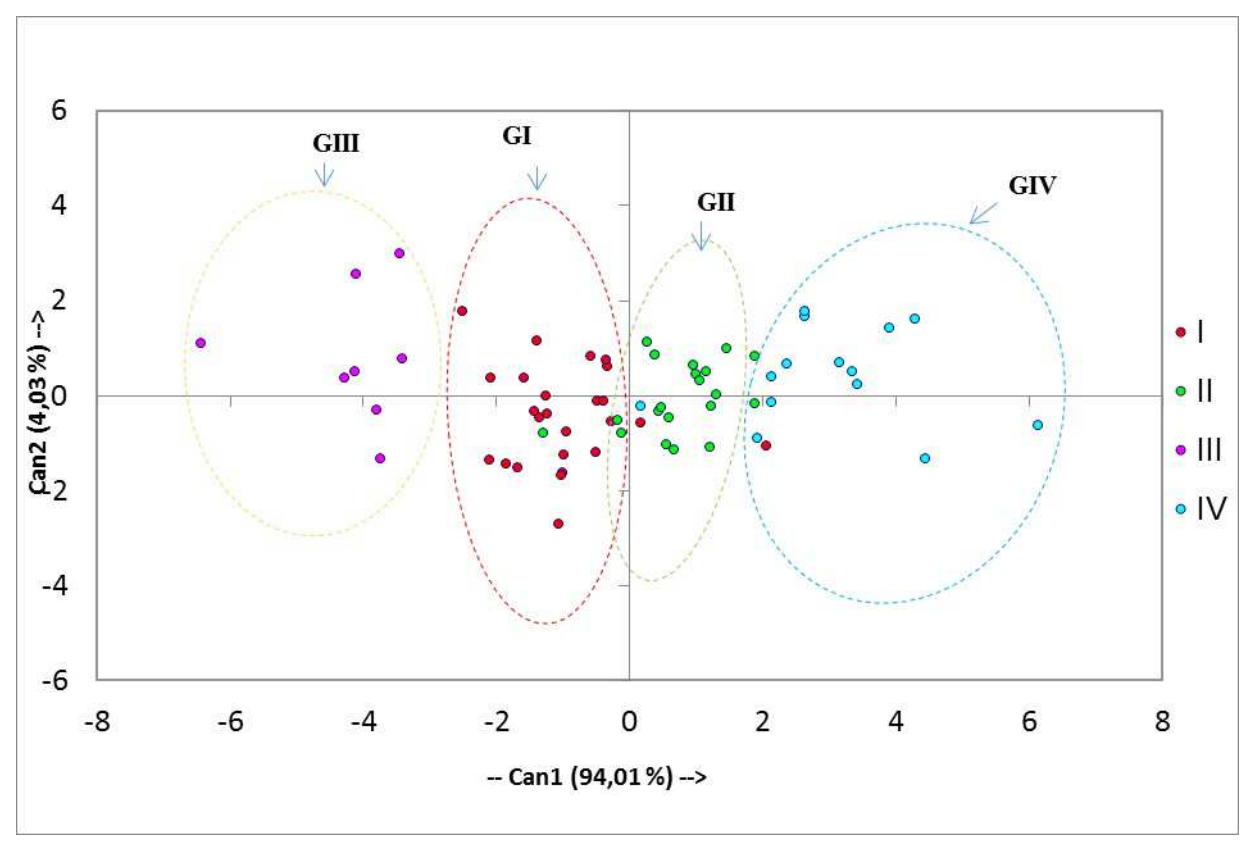

Figure 3 : Projection des groupes dans le plan formé par les deux premiers axes canoniques. 


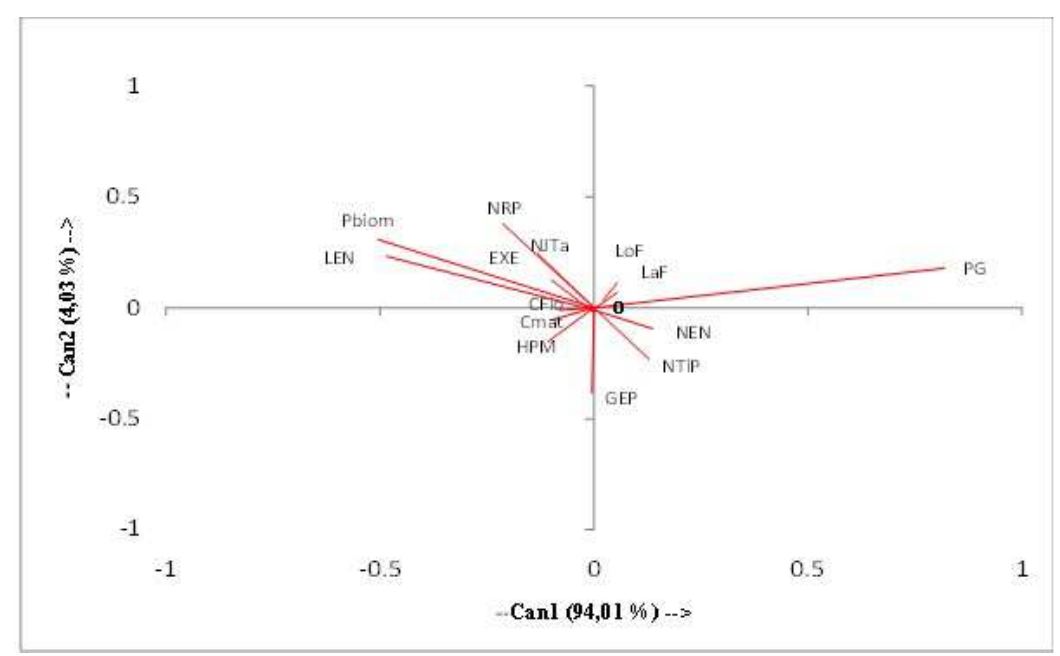

Figure 4: Projection des variables mesurés dans le plan formé par les deux premiers axes canoniques.

Aussi, la variation de ces caractères est sans nul doute liée à l'expression d'un ou plusieurs gènes dont l'identification pourrait être faite par l'analyse moleculaire. Par ailleurs, les individus ayant la couleur marron de leur graine non decortiquée ont été observés uniquement dans la region de Dosso plus particulierement dans les villages de Oumgoua Doutchi, Birni Fallao, Sandiday et Wassangou.

L'étude des accessions locales du fonio a permis d'apprécier la diversité de l'espèce au Niger. Les amplitudes de variation élevées observées pour un nombre significatif de caractères indiquent la présence d'une forte hétérogénéité au sein des accessions locales $\mathrm{du}$ fonio. La dispersion et les moyennes obtenues pour chaque caractère quantitatif sont nettement supérieures à celles observées dans des études similaires réalisées par Adoukounou-Sagbadja et al. (2007b) au Benin sur 122 accessions originaires de l'Afrique de l'ouest, par Kanfany (2009) au Sénégal à partir d'un cultivar local évalué dans des conditions de fertilisation differentes, par Dansi et al. (2010) au Nord-Ouest du Benin.

L'ensemble de ces résultats témoignent de l'existence d'une diversité importante au sein de l'espèce. Cette importante variabilité phénotypique observée pourrait résulter de l'expression d'une forte hétérogénéité génotypique qui restera à confirmer par les analyses moleculaires, mais aussi de l'influence des facteurs environnementaux.

En effet, des travaux ont montré que la longueur du jour ou photoperiodisme entraîne des effets variables sur le développement végétatif et physiologique du fonio (Aliero et Morakinyo, 2005). L'étude des corrélations a montré l'existence d'une rélation nette entre les caractères morphologiques végétatifs et le rendement en grains des plantes.

C'est ainsi que les accessions précoces dont la durée du cycle est inferieur ou égale à 90 JAS donnent les meilleurs rendements en grains alors que les accessions tardives à cycle supérieur à 90 JAS et qui ont par ailleurs des valeurs élevées de biomasse sèche et de longueur des entrenoeuds ont les plus faibles rendements en graines.

Cela pourait s'expliquer soit par la perte des grains lié à leur poids à maturité sur le panicule de la plante, soit par une concentration élévée de l'azote au niveau de la plante. 
Tableau 3: Analyse des 14 caractères quantitatifs mesurés pour les 67 accessions du fonio du Niger.

\begin{tabular}{ccccccc}
\hline $\mathbf{N}^{\circ}$ & Caractères & Moyennes & Ecart types & Minimum & Maximum & CV (\%) \\
\hline 1 & EXE & 8,84 & 0,99 & 6,90 & 12,40 & 11,2 \\
2 & LoF & 10,79 & 0,63 & 8,60 & 12,20 & 5,8 \\
3 & LaF & 0,51 & 0,07 & 0,40 & 1,00 & 14,7 \\
4 & NEN & 6,96 & 0,64 & 5,60 & 8,20 & 9,1 \\
5 & LEN & 6,02 & 1,12 & 4,15 & 9,75 & 18,5 \\
6 & NJT & 24,69 & 1,05 & 21,83 & 26,67 & 4,3 \\
7 & NTP & 10,91 & 1,69 & 8,10 & 14,53 & 15,5 \\
8 & FLO & 62,96 & 1,07 & 59,75 & 65,42 & 1,7 \\
9 & MAT & 90,66 & 1,55 & 86,17 & 94,33 & 1,7 \\
10 & NdP & 3,45 & 0,14 & 3,12 & 3,88 & 4,1 \\
11 & HPM & 70,72 & 2,62 & 63,71 & 78,82 & 3,7 \\
12 & PBM & 7887,49 & 1117,24 & 5766,62 & 13072,04 & 14,2 \\
13 & PGR & 954,47 & 181,63 & 604,30 & 1362,10 & 19,0 \\
14 & GEP & 2,58 & 0,72 & 2,00 & 4,00 & 27,9 \\
\hline
\end{tabular}

Les accessions précoces identifiées dans cette étude sont qualifiées d'extraprécoces à hatives selon les critères proposés par Portères (1976). Cette précocité permet d'envisager plus d'une culture par an.

Les accessions tardives au rendement en biomasse plus élévé peuvent être selectionnées à de fins alimentaires pour les betails grâce à leurs forte productivité en fourrage. Par ailleurs, nos résultats sont en conformité avec ceux de Ouédraogo et al. (2008) qui ont rapporté qu'au Burkina-Faso, les accessions tardives donnaient les plus faibles rendements en graines.

Les corrélations constituent un outil indispensable pour les améliorateurs dans le choix des caractères à intégrer dans les programmes de sélection.

Les analyses multivariées à travers la CAH et l'AFD ont montré que la variabilité de nos accessions est structurée en quatre groupes sur la base des caractères rendement en graine et en biomasse sèche par hectare Il ressort, de la forte représentativité des deux premiers axes canoniques de l'AFD $(98,04 \%)$, l'existence d'une forte organisation génotypique et phénotypique du matériel étudié.

Les dissemblances morphologiques et phénologiques observées entre les différents groupes phénotypiques suggèrent que les accessions sont maintenues sous des processus évolutifs très différents dans leur agroécosystème respectif.

Les agroécosystèmes sont susceptibles d'exercer des pressions sélectives très variables sur les génotypes (Sadiki et Jarvis, 2005) ainsi que les pressions anthropiques (Robert et al., 2004). En effet, le mode de gestion des semences par les agriculteurs tels que les tris sélectifs, les techniques post recolte de la culture et les pratiques agricoles conduisent à une sélection entraînant le maintien, voire la création d'une diversité phénotypique importante (Robert et al., 2005). 
Tableau 4: Résultats de l'analyse de variance sur les 16 paramètres quantitatifs.

\begin{tabular}{|c|c|c|c|c|c|c|c|c|c|c|c|c|c|c|c|}
\hline \multirow{2}{*}{ Source de variation } & \multirow[t]{2}{*}{ ddl } & NJT & NTP & FLO & MAT & NRP & HPM & PBM & PGR & EXE & LoF & LaF & NEN & LEN & GEP \\
\hline & & $\mathbf{F}$ & $\mathbf{F}$ & $\mathbf{F}$ & $\mathbf{F}$ & $\mathbf{F}$ & $\mathbf{F}$ & $\mathbf{F}$ & $\mathbf{F}$ & $\mathbf{F}$ & $\mathbf{F}$ & $\mathbf{F}$ & $\mathbf{F}$ & $\mathbf{F}$ & $\mathbf{F}$ \\
\hline Accessions & 66 & $0,27^{\mathrm{ns}}$ & $0,69^{\text {ns }}$ & $0,15^{*}$ & $0,53^{*}$ & $0,89^{\mathrm{ns}}$ & $1,36^{*}$ & $1,10^{* * *}$ & $1,54^{* * *}$ & $23,26^{* * * *}$ & $22,48^{* * * *}$ & $32,65^{* * *}$ & $26,48^{* * * *}$ & $29,60^{* * *}$ & $26,51^{* * *}$ \\
\hline localité & 1 & $20,51^{* * *}$ & $353,93^{* * *}$ & $873,80^{* * *}$ & $450,41^{* * * *}$ & $2,23^{\mathrm{ns}}$ & $0,47^{\text {ns }}$ & $488,81^{* * * *}$ & $9,07^{* *}$ & $0,04^{\mathrm{ns}}$ & $0,20^{\mathrm{ns}}$ & $0,01^{\text {ns }}$ & $0,35^{\mathrm{ns}}$ & $0,28^{\mathrm{ns}}$ & $0,02^{\mathrm{ns}}$ \\
\hline année & 1 & $224,41^{* * *}$ & $86,56^{* * *}$ & $424,41^{* * *}$ & $421,71^{* * * *}$ & $3,25^{\mathrm{ns}}$ & $7,87^{* *}$ & $411,80^{* * * *}$ & $573,56^{* * *}$ & $0,04^{\mathrm{ns}}$ & $0,20^{\mathrm{ns}}$ & $0,01^{\mathrm{ns}}$ & $0,35^{\mathrm{ns}}$ & $0,28^{\mathrm{ns}}$ & $0,00^{\mathrm{ns}}$ \\
\hline Bloc & 2 & $3,57^{*}$ & $9,84^{* * *}$ & $0,09^{\mathrm{ns}}$ & $0,08^{\mathrm{ns}}$ & $1,32^{\mathrm{ns}}$ & $7,29^{* *}$ & $0,01^{\mathrm{ns}}$ & $9,25^{* * *}$ & $0,13^{\mathrm{ns}}$ & $0,09^{\mathrm{ns}}$ & $0,02^{\mathrm{ns}}$ & $0,16^{\mathrm{ns}}$ & $0,39^{\mathrm{ns}}$ & $0,00^{\mathrm{ns}}$ \\
\hline Accessions*année & 66 & $0,35^{\mathrm{ns}}$ & $0,97^{\mathrm{ns}}$ & $0,18^{\mathrm{ns}}$ & $0,44^{\mathrm{ns}}$ & $0,79^{\mathrm{ns}}$ & $1,02^{\mathrm{ns}}$ & $0,66^{\mathrm{ns}}$ & $1,55^{* *}$ & $6,05^{* * * *}$ & $6,57^{* * * *}$ & $6,15^{* * *}$ & $6,49^{* * *}$ & $6,03^{* * *}$ & $6,74^{* * *}$ \\
\hline Accessions*localité & 66 & $0,27^{\mathrm{ns}}$ & $0,63^{\mathrm{ns}}$ & $0,17^{\mathrm{ns}}$ & $0,65^{\mathrm{ns}}$ & $0,74^{\mathrm{ns}}$ & $1,30^{\mathrm{ns}}$ & $1,11^{\mathrm{ns}}$ & $1,19^{* *}$ & $6,05^{* * *}$ & $6,57^{* * * *}$ & $6,15^{* * *}$ & $6,49^{* * * *}$ & $6,03^{* * *}$ & $6,59^{* * *}$ \\
\hline Accessions*Bloc & 132 & $0,23^{\mathrm{ns}}$ & $0,88^{\mathrm{ns}}$ & $0,07^{\mathrm{ns}}$ & $0,21^{\mathrm{ns}}$ & $0,81^{\mathrm{ns}}$ & $1,04^{\mathrm{ns}}$ & $1,36^{*}$ & $0,86^{\mathrm{ns}}$ & $0,04^{\mathrm{ns}}$ & $0,04^{\mathrm{ns}}$ & $0,01^{\mathrm{ns}}$ & $0,05^{\mathrm{ns}}$ & $0,15^{\mathrm{ns}}$ & $0,03^{\mathrm{ns}}$ \\
\hline
\end{tabular}


Tableau 5: Corrélation de Pearson entre variables initiales.

\begin{tabular}{|c|c|c|c|c|c|c|c|c|c|c|c|c|c|}
\hline & EXE & LoF & LaF & NEN & LEN & NJT & NTP & FLO & MAT & NdP & HPM & PBM & PGR \\
\hline EXE & 1.0000 & 0.2456 & -0.0081 & -0.0933 & 0.0962 & 0.0313 & -0.0046 & 0.0112 & 0.1531 & -0.0495 & -0.2063 & -0.0218 & -0.1374 \\
\hline LoF & & 1.0000 & 0.1413 & -0.2137 & -0.0369 & -0.1228 & 0.1566 & -0.0859 & 0.0282 & -0.0078 & 0.0776 & 0.0765 & 0.0899 \\
\hline $\mathrm{LaF}$ & & & 1.0000 & -0.1350 & -0.0425 & 0.1442 & 0.1833 & 0.1270 & -0.0523 & -0.1651 & 0.0717 & 0.1269 & 0.2087 \\
\hline NEN & & & & 1.0000 & -0.0735 & 0.0025 & 0.0567 & 0.0733 & -0.2049 & 0.0909 & 0.1425 & 0.1947 & 0.0065 \\
\hline LEN & & & & & 1.0000 & -0.0374 & 0.0021 & 0.1462 & 0.3838 & 0.0035 & -0.2726 & 0.5358* & $-0.5048 *$ \\
\hline NJT & & & & & & 1.0000 & -0.0575 & 0.1180 & 0.1679 & -0.0364 & 0.1340 & 0.0232 & -0.1677 \\
\hline NTP & & & & & & & 1.0000 & -0.0507 & -0.2521 & 0.0411 & 0.0554 & 0.0155 & $0.5358 *$ \\
\hline FLO & & & & & & & & 1.0000 & 0.2010 & 0.0703 & 0.1051 & 0.0934 & -0.1067 \\
\hline MAT & & & & & & & & & 1.0000 & -0.2419 & 0.0011 & -0.2883 & -0.2394 \\
\hline $\mathrm{NdP}$ & & & & & & & & & & 1.0000 & 0.0241 & 0.0639 & -0.1526 \\
\hline HPM & & & & & & & & & & & 1.0000 & 0.0261 & -0.1830 \\
\hline PBM & & & & & & & & & & & & 1.0000 & $-0.8186 *$ \\
\hline PGR & & & & & & & & & & & & & 1.0000 \\
\hline
\end{tabular}

FLO : cycle de floraison; MAT : cycle de maturité a 50\% ; HPM : hauteur de la plante à maturité ; NJT : Nombre des jours $50 \%$ Tallage ; NTP : Nombre de talles par plante ; NRP: Nombre du racème par panicule; PGR : poids des graines par hectare; GEP : Groupement des épillets sur les pédicelles au niveau des racèmes ; PBM : Poids de la biomasse sèche par hectare : EXE : Exertion de la panicule ; LoF : Longueur des feuilles; LaF: Largeur des feuilles; NEN : Nombre d'entrenœud; LEN : Longueur des entrenœuds, $*$ coefficient de corrélation significative au seuil ( $\alpha=0,05)$. 
Tableau 6: Résultats des tests univarié et multivarié du pouvoir discriminant des groupes par les variables : Valeurs de $\mathrm{R}^{2}$ et significativité.

\begin{tabular}{lccc}
\hline Variables & $\mathbf{R}^{\mathbf{2}}$ & $\mathbf{F}(\mathbf{3})^{*}$ & Prob \\
\hline NJT & 0,30 & 0,778 & 0,511 \\
NTP & 0,50 & 0,587 & 0,626 \\
FLO & 0,10 & 0,305 & 0,822 \\
MAT & 0,53 & 0,334 & 0,801 \\
NRP & 0,59 & 1,434 & 0,241 \\
HPM & 0,23 & 0,383 & 0,766 \\
PBM & 0,96 & 6,146 & 0,001 \\
PGR & 0,92 & 25,939 & $<0,0001$ \\
GEP & 0,60 & 0,673 & 0,571 \\
EXE & 0,005 & 0,110 & 0,953 \\
LoF & 0,03 & 0,570 & 0,635 \\
LaF & 0,04 & 0,970 & 0,411 \\
NEN & 0,08 & 1,900 & 0,139 \\
LEN & 0,70 & 4,530 & 0,006 \\
Statistique de Wilks' Lambda & & $36,(\mathrm{ddl}=150)$ & $<0,0001$ \\
\hline
\end{tabular}

Tableau 7 : Proportions d'informations concentrées sur les axes canoniques, corrélations canoniques et significativité des axes canoniques (Test de Bartlett).

\begin{tabular}{lcccccc}
\hline Axes & Proportions & $\begin{array}{c}\text { Proportions } \\
\text { cumulées }\end{array}$ & $\mathbf{R}_{\text {can }^{2}}$ & $\mathbf{K h i}^{\mathbf{2}}$ & ddl & Prob \\
\hline $\mathbf{1}$ & 0,9401 & 0,9401 & 0,910 & 119,566 & 33 & 0,0001 \\
$\mathbf{2}$ & 0,0403 & 0,9804 & 0,413 & 16,566 & 20 & 0,6810 \\
$\mathbf{3}$ & 0.0196 & 1.0000 & 0.302 & 5.598 & 9 & 0.7790 \\
\hline
\end{tabular}

Ces facteurs évolutifs pourraient être les causes de la nette différenciation agromorphologique et phénologique observées entre les accessions. Cette évolution constante, influencée par les facteurs pédologiques, climatiques et anthropiques dans les différents agroécosystèmes, donne aux accessions des caractéristiques qui leurs sont propres (Azam-Ali et al., 2001). La présence du groupe de diversité GI et GII avec des caractéristiques intermédiaires entre les accessions précoces et tardives pourrait être le résultat d'un processus adaptatif.

En effet, la structuration de la diversité des accessions du fonio du Niger n'a pas permis de définir une organisation communale, régionale ou spatiale à partir des caractères agromorphologiques quantitatifs évalués car les différents groupes définis par les analyses multivariées renferment des accessions provenant de chaque zone de collecte (Communes ou Regions).

La grande variabilité observée dans cette étude pourrait être due à des échanges des semences de ces différentes accessions cultivées entre le producteur d'un village à un autre village voisin ou éloigné. Ce type d'observation qui montre la forte variabilité intra-accession et inter-accession a été décrit par plusieurs auteurs chez différentes espèces cultivées en Afrique de l'Ouest (Rouamba et al., 2001; Demol et al., 2002 ; Barnaud et al., 2007; Bakasso, 2010 ; Antoine et al., 2012). 
Tableau 8 : Caractéristiques des groupes: valeurs moyennes et écarts-types des paramètres par groupe, valeur de la statistique de Newmann Keuls et leur significativité.

\begin{tabular}{|c|c|c|c|c|c|c|c|c|c|c|c|c|c|}
\hline \multirow{2}{*}{ Variables } & \multicolumn{2}{|c|}{ GrpI (N=19) } & \multicolumn{2}{|c|}{ Grp II (N=11) } & \multicolumn{2}{|c|}{ Grp III (N=8) } & \multicolumn{2}{|c|}{ Grp IV (N=29) } & \multicolumn{2}{|c|}{ Global } & \multicolumn{2}{|c|}{ Statistique du test } & \multirow[b]{2}{*}{$\mathrm{CV}(\%)$} \\
\hline & $\mathbf{m}$ & $\mathbf{s}$ & $\mathbf{m}$ & $\mathbf{s}$ & $\mathbf{m}$ & $\mathbf{s}$ & $\mathbf{m}$ & $\mathbf{s}$ & $\mathbf{m}$ & $\mathbf{s}$ & $\mathbf{F}$ & Prob & \\
\hline NJT & $24,62^{\mathrm{a}}$ & 1,19 & $24,68^{\mathrm{a}}$ & 0,95 & $25,14^{\mathrm{a}}$ & 0,43 & $24,59^{\mathrm{a}}$ & 1,19 & $24,76^{\mathrm{a}}$ & 0,26 & 0,778 & 0.655 & 4,3 \\
\hline NTP & $10,98^{\mathrm{a}}$ & 1,59 & $10,93^{\mathrm{a}}$ & 1,86 & $10,23^{\mathrm{a}}$ & 0,89 & $11,05^{\mathrm{a}}$ & 1,97 & $10,80^{\mathrm{a}}$ & 0,38 & 0,587 & 0,688 & 5,5 \\
\hline FLO & $63,00^{\mathrm{a}}$ & 0,93 & $63,00^{\mathrm{a}}$ & 1,24 & $63,10^{\mathrm{a}}$ & 0,98 & $62,76^{\mathrm{a}}$ & 1,19 & $62,97^{\mathrm{a}}$ & 0,14 & 0,305 & 0,874 & 1,7 \\
\hline MAT & $90,85^{\mathrm{a}}$ & 1,88 & $88,31^{\mathrm{a}}$ & 1,64 & $90,88^{\mathrm{a}}$ & 1,05 & $86,69^{\mathrm{a}}$ & 0,93 & $90,68^{\mathrm{a}}$ & 0,26 & 0,334 & 0,673 & 1,7 \\
\hline NRP & $3,43^{\mathrm{a}}$ & 0,14 & $3,44^{\mathrm{a}}$ & 0,14 & $3,54^{\mathrm{a}}$ & 0,13 & $3,43^{\mathrm{a}}$ & 0,14 & $3,46^{\mathrm{a}}$ & 0,05 & 1,434 & 0.254 & 4,1 \\
\hline HPM & $70,94^{\mathrm{a}}$ & 2,68 & $70,78^{\mathrm{a}}$ & 3,06 & $70,90^{\mathrm{a}}$ & 1,70 & $70,12^{\mathrm{a}}$ & 2,41 & $70,69^{\mathrm{a}}$ & 0,38 & 0,383 & 0.816 & 3,7 \\
\hline PBM & $7972,7^{\mathrm{b}}$ & 912,84 & $7696,61^{b}$ & 806,02 & $9148,07^{\mathrm{a}}$ & 1797,08 & $7287,66^{\mathrm{b}}$ & 821,48 & $8026,26^{\mathrm{b}}$ & 799,07 & 6,146 & 0,001 & 27,9 \\
\hline PGR & $882,72^{c}$ & 130,99 & $993,13^{\mathrm{b}}$ & 113,76 & $716,80^{\mathrm{d}}$ & 113,91 & $1163,17^{\mathrm{a}}$ & 126,69 & $938,96^{\mathrm{c}}$ & 187,73 & 25,939 & $<0,0001$ & 19,0 \\
\hline GEP & $2,72^{\mathrm{a}}$ & 0,79 & $2,55^{\mathrm{a}}$ & 0,76 & $2,38^{a}$ & 0,52 & $2,50^{\mathrm{a}}$ & 0,65 & $2,54^{\mathrm{a}}$ & 0,14 & 0,673 & 0.625 & 14,2 \\
\hline EXE & $8,816^{\mathrm{a}}$ & 0,644 & $8,982^{\mathrm{a}}$ & 0,665 & $8,725^{\mathrm{a}}$ & 0,838 & $8,831^{\mathrm{a}}$ & 1,301 & $8,84^{\mathrm{a}}$ & 0,22 & 0,110 & 0.3687 & 11,2 \\
\hline LoF & $10,67^{\mathrm{a}}$ & 0,565 & $10,71^{\mathrm{a}}$ & 0,509 & $10,75^{\mathrm{a}}$ & 1,090 & $10,90^{\mathrm{a}}$ & 0,559 & $10,76^{\mathrm{a}}$ & 0,19 & 0,570 & 0.139 & 5,8 \\
\hline $\mathbf{L a F}$ & $0,516^{\mathrm{a}}$ & 0,121 & $0,489^{\mathrm{a}}$ & 0,045 & $0,538^{\mathrm{a}}$ & 0,052 & $0,503^{\mathrm{a}}$ & 0,042 & $0,51^{\mathrm{a}}$ & 0,02 & 0,970 & 0.081 & 4,7 \\
\hline NEN & $6,808^{\mathrm{a}}$ & 0,625 & $7,055^{\mathrm{a}}$ & 0,587 & $7,406^{\mathrm{a}}$ & 0,577 & $6,909^{\mathrm{a}}$ & 0,645 & $7,04^{\mathrm{a}}$ & 0,16 & 1,900 & 0.813 & 9,1 \\
\hline LEN & $5,674^{\mathrm{b}}$ & 1,144 & $5,991^{b}$ & 0,811 & $7,212^{\mathrm{a}}$ & 0,698 & $5,816^{\mathrm{b}}$ & 1,101 & $5,92^{\mathrm{b}}$ & 0,24 & 4,530 & 0,005 & 18,5 \\
\hline
\end{tabular}

FLO : cycle de floraison; MAT : cycle de maturité a 50\% ; HPM : hauteur de la plante à maturité ; NJT : Nombre des jours 50\% Tallage ; NTP : Nombre de talles par plante ; NRP: Nombre du racème par panicule; PGR : poids des graines par hectare ; GEP : Groupement des épillets sur les pédicelles au niveau des racèmes ; PBM: Poids de la biomasse sèche par hectare ; EXE : Exertion de la panicule ; LoF : Longueur des feuilles ; LaF : Largeur des feuilles ; NEN : Nombre d'entrenœud ; LEN : Longueur des entrenœuds. Sur chaque ligne les valeurs accompagnées par des lettres différentes sont significativement différents au seuil de probabilité $(\alpha=0,05)$. 


\section{Conclusion}

La variabilité observée à l'intérieur des accessions étudiées constitue un argument de choix pour la conservation en milieu paysan des cultivars traditionnelles du fonio du Niger. Cette variabilité importante au sein de l'espèce pourrait être favorisée par le mode de reproduction qui reste à être confirmé par les analyses moléculaires.

La place des accessions locales dans les ressources végétales des plantes cultivées n'étant plus à démontrer, une attention particulière doit être accordée aux régions qui assurent la pérennité de cette source de variabilité. Les caractères analysés peuvent ainsi constituer des critères de base pour différencier les accessions des autres régions du Niger et servir dans des études de diversité morphologique du fonio en Afrique de l'Ouest. Ces résultats montrent la nécessité de tester l'approche génétique par l'utilisation de marqueurs moléculaires afin de mieux connaître la diversité génétique globale ainsi que le régime de reproduction. Cette diversité comparée avec celle identifée par la perception des paysans, mais aussi à la diversité obtenue à l'issue de l'évaluation phénotypique des accessions collectées au Niger, permet une utilisation rationnelle de cette ressource dans les programmes de l'amélioration du fonio du Niger.

\section{REMERCIEMENTS}

Cette étude a été réalisée grâce à un financement de l'Université Abdou Moumouni. Nous remercions l'Institut National de Recherche Agronomique du Niger (INRAN) pour nous avoir autorisé à réaliser notre expérimentation dans leurs stations de recherche de Tarna et de Tara et l'IRD pour son appui logistique.

\section{REFERENCES}

Abdelkader M.S. 2012. Etude de l'effet de la gestion intégrée de la fertilité du sol sur la production de maïs (Zea mays). Mémoire de fin de cycle présentée à la Faculté des Sciences et Techniques de l'Université de Maradi pour l'obtention du diplôme de Master II, Université de Maradi, Maradi, Niger, p.71.

Adoukonou-Sagbadja H, Dansi A, Voudouhe R. 2006. Indigenous knowledge and traditional conservation of fonio millet (Digitaria exilis, Digitaria iburua) in Togo. Biodiversity and Conservation, 15: 2379-2395.

Adoukonou-Sabadja H, Schubert V, Dansi A, Jovtchev G, Meister A, Pistrick K, Akpagna K, Friedt W. 2007a. Flow cytometric analysis reveals different nuclear DNA contents in cultivated fonio (Digitaria spp.) and some wild relatives from West Africa. Plant Systematics and Evolution, 267: 163176.

Adoukonou-Sagbadja H, Wagner C, Dansi A. 2007b. Genetic diversity and population differentiation of traditional fonio millet (Digitaria spp.) landraces from different agro-ecological zones of West Africa. Theoretical and Applied Genetics, 7: 917-931.

Adoukonou-Sagbadja H, Wagner C, Ordon F, Freidt W. 2010. Reproductive system and molecular phylogenetic relationships of fonio millets (Digitaria spp. Poaceae) with some polyploidy wild relatives. Tropical Plant Biology, 10: $240-251$.

Ahmadi N, Becquer T, Larroque C, Arnaud M. 1988. Variabilité génétique du riz (Oryza sativa L.) à Madagascar. Agron. Trop, 43: 209-221. 
Aliero AA, Morakinyo JA. 2005. Photoperiodism in Digitaria exilis. African Journal of Biotechnology, 4: 241-243.

Antoine AM, Adoukonou-Sagbadja H, Rollande AD, Corneille A, Clément A. 2012: Impacts des pratiques culturales sur l'organisation génétique des sorghos cultivés par les Lokpa au NordOuest du Bénin révélés par les marqueurs SSRs. Journal of Applied Biosciences, 60: 4394-4409.

Azam-ali SN, Sesay A, Karikari SK, Massawe FJ, Aguilar-manjarrez J, Bannayan M, Hampson KJ. 2001. Assessing the potential of an underutilized crop - A case study using bambara groundnut. Experimental Agriculture, 37: 433-472.

Bakasso Y. 2010. Ressources génétiques des roselles (Hibiscus sabdariffa L.) du Niger : évaluations agromorphologique et génétique. Thèse de Doctorat d'état, université de Niamey, Niamey, p .139.

Bama J. 1999. Intérêt du fonio dans l'alimentation des diabétiques: Thèse de doctorat, Université de Ouagadougou, Ouagadougou, p. 120.

Barnaud A. 2007. Savoirs, pratiques et dynamique de la diversité génétique : le sorgho (Sorghum bicolor ssp. bicolor) chez les Duupa du Nord Cameroun. Thèse de doctorat, université de Montpellier II, Montpellier, p. 282.

Brown AHD, Munday J. 1982. Populationgenetic structure and optimal sampling of landraces of barley from Iran. Genetica, 58: 85-96.

Brush SB. 1995. In situ conservation of landraces in centers of diversity. Crop Sci, p. 35.

Chantereau J, Arnaud M, Ollitrault P, Nabayaogo P, Noyer JL. 1989. Étude de la diversité morphophysiologique et classification des sorghos cultivés. Agron. Trop, 44: 223-232.

Cherifi K, Boussaïd M, Marrakchi M. 1993. Diversité génétiques de quelques populations naturelles de Medicago ciliaris (L) Krock et de Medicago intertexta (L) Mill. Analyse de la variabilité morphologique. Agronomie (Elsevier/INRA), 13: 895-908.

Cruz JF. 2004. Fonio a small grain with potential. LEISA, magazine on low external input and sustainable agriculture valuing Crop diversity, 1 : 16-17.

Cruz JF, beavogui F, Drame D. 2011. Le Fonio, une Céréale Africaine. Collection Agricultures tropicales en poche. Editions. Quae / Cta / Presses agronomiques de Gembloux:Versailles, France; 175 p.

Dansi A, Adoukonou-Sagbadja H, Vodouhè R. 2010. Diversity, conservation and relatedwild species of fonio millet (Digitaria spp.) in the northwest of Benin. Genetic Resources and Crop. Evolution, 57: 827-839.

Demol J, Baudouin JP, Louant BP, Marechal R, Mergeai G, Otoul E. 2002. Amélioration des plantes. Application aux principales espèces cultivées en régions tropicales. Presses agronomiques de Gembloux, Gembloux, Belgique, $582 \mathrm{p}$.

Djè Y, Ater M, Lefèbvre C, Vekemans X. 1998. Patterns of morphological and allozyme variation in sorghum landrace of northwestern Morocco. Genet. Res, Crop. Evol., 45: 541-548.

Doebley J, Goodman M, Stuber C. 1985. Isozyme variation in the races of maize from Mexico. Amer. J. Bot., 75: 629639. 
Ezekiel CN, Sulyok M, Warth B. 2012. Multimicrobial metabolites in fonio millet (acha) and sesame seeds in Plateau State, Nigeria. European Food Research and Technology, 235: 285293.

Fofana B, Vekemans X, du Jardin P, Baudoin JP. 1997. Genetic diversity in Lima bean (Phaseolus lunatus L.) as revealed by RAPD markers. Euphytica, 95: 157165.

Frankel O, Brown AHD, Burdon JJ. 1995. The Conservation of Plant Biodiversity. Cambridge University Press: New York, USA; 299 p.

Harlan JR. 1975. Our vanishing genetic resources. Science, 188: 618-621.

Jideani IA. 2012. Digitaria exilis (acha/fonio), Digitaria iburua (iburu/fonio) and Eleusine coracana (tamba/finger millet)-Nonconventional cereal grains with potentials. Scientific Research and Essays, 45: 3834-3843.

Jideani IA, Jideani VA. 2010. Development on the cereal grains Digitaria exilis (acha) and Digitaria iburua. Journal of Food Science and Technology, 3: 251259.

Kanfany MG. 2009. Effets de la fertilisation organo minérale sur la croissance et le rendement du fonio (Digitaria exilis Stapf) en Casamance et au Sénégal Oriental. Memoire de Diplome d'Etude Approfondie (DEA), Université Cheik Anta Diop. 35.p.

Maquet A, Zoro BI, Delvaux M, Wathelet B, Baudoin JP. 1997. Genetic structure of a Lima bean base collection using allozyme markers. Theor. Appl. Genet, 95: 980-991.
McLACHLAN GJ. 1992. Discriminant Analysis and Statistical Pattern Recognition. Wiley: New-York; $526 \mathrm{p}$ Ouédraogo M, Ouédraogo JT, Tignéré JB, Balma D, Dabiré CB, Konaté G. 2008. Characterization and evaluations of accessions of bambara groundnut (Vigna subterranea (L.) Verdcourt) from Burkina Faso. Sciences et Nature, 5(2): 191-197.

Oumarou DD. 2012. Le refus de la culture du fonio dans le village de Tombon-Dogo, Commune Rurale de guéchémé (Département de Tibiri). Thèse Mémoire présentée à la Faculté des Lettres et Sciences Humaines de l'Université de Niamey pour obtenir le grade de Maitrise ès lettres/option: Sociologie Rurale, 59p.

Poilecot P. 1999: Les Poaceae du Niger: Mémoires de botanique systématique. Boissiera , 56(1) : 448-469 Africain. Agron Trop, 1: 589-592.

Porteres R. 1976. African cereals: Eleusine, Fonio, Black Fonio, Teff, Brachiara, Paspalum, Pennisetum, and African Rice. In The Origins of African Plant Domestication, Harlan J, de Wet JMJ, Stemler ABL (eds). The Hague: Mouton.

Portères R. 1946: L'aire culturale du Digitaria iburua Stapf, céréale mineure de l'ouest Africain. Agron Trop, 1: 589-592.

Robert T, Luxereau A, Mariac C, Ali K, Allinne C, Bani J, Beidari Y, Bezançon G, Gayeux S, Couturon E, Dedieu V, Sadou M, Seydou M, Seyni O, Tidjani M, Sarr A. 2004. Gestion de la diversité en milieu paysan : influence de facteurs anthropiques et des flux de gènes sur la variabilité génétique des formes cultivées et spontanées du mil 
(Pennisetum glaucum ssp. glaucum) dans deux localités du Niger, Actes du 4 e colloque national. Le patrimoine génétique : la diversité et la ressource. La Châtre, 14-16 octobre 2002. Bureau des ressources génétiques 2004.

Robert T, Mariac C, Allinne C, Ali K, Beidari Y, Bezançon G, Couturon E, Moussa D, Sasou MS, Seydou M, Seyni O, Tidjani M, Luxereau A. 2005. Gestion des semences et dynamiques des introgressions entre variétés cultivées et entre formes domestiques et spontanées des mils (Pennisetum glaucum ssp. glaucum) au Sud-Niger. Les Actes du BRG, 5 e colloque national. Un dialogue pour la diversité. Lyon, 3-4-5 novembre 2004.

Rouamba A, Sandmeier M, Sarr A, Ricroch A. 2001. Allozyme variation within and among populations of onion (Allium cepa L.) from West Africa. Theoretical and Applied Genetics, 103: 855-861.

Sadiki M, Jarvis D. 2005. Conservation in situ de la diversité génétique des cultures par sa gestion à la ferme dans les agroécosystèmes marocains. Les Actes du BRG, 5: 445-464.

SAS. 2010. Using JMP 9. SAS Institute Inc., Cary: NC, USA ; 599.

Sivakumar M. V. K., Maidoukia A. \& Stern R. D .1993. Agroclimatology of West Africa: Niger. Information Bulletin $\mathrm{n}^{\circ}$ 5, ICRISAT; 108.

Vall E, Andrieu N, Beavogui F, Sogodogo D. 2011. Les cultures de soudure comme stratégie de lutte contre 'insécurité alimentaire saisonnière en Afrique de l'Ouest : le cas du fonio Digitaria exilis Stapf). Cah Agric, 20: 294-300. 